\title{
Population based approaches to computational musculoskeletal modelling
}

\author{
Justin Fernandez ${ }^{1} \cdot$ Alex Dickinson $^{2} \cdot$ Peter Hunter $^{1}$
}

Published online: 28 July 2020

(c) Springer-Verlag GmbH Germany, part of Springer Nature 2020

\section{Review:}

- Machine learning methods to support personalized neuromusculoskeletal modelling (Saxby et al. 2020)

\section{Population Anatomic Analysis and Model Reconstruction:}

- The Morphology of the Human Mandible: A Computational Modelling Study (Vallabh et al. 2019)

- Statistical shape modelling of the first carpometacarpal joint reveals high variation in morphology (Rusli and Kedgley 2019)

- Relating foot shape and plantar pressure function in shod and barefoot populations (Mei et al. 2019)

- Best methods and data to reconstruct paediatric lower limb bones for musculoskeletal modelling (Davico et al. 2019)

- Rapid Muscle Volume Prediction using Anthropometric Measurements and Population-Derived Statistical Models (Yeung et al. 2019)

Mechanobiology: (bone remodelling and multiscale analysis):

- Influence of femoral external shape on internal architecture and fracture risk (Villette et al. 2019)

- Three-dimensional rendering of trabecular bone microarchitecture using a probabilistic approach (Kirby et al. 2020)

Justin Fernandez

j.fernandez@auckland.ac.nz

1 Auckland Bioengineering Institute, University of Auckland, Auckland, New Zealand

2 University of Southampton, Southampton, UK
Design and Assessment of Standardised Orthopaedic Devices:

- Computational efficient method for assessing the influence of surgical variability on primary stability of a contemporary femoral stem in a cohort of subjects (Al-Dirini et al. 2019)

- Impact of alignment and kinematic variation on resistive movement and dislocation propensity for THA with lipped and neutral liners (Huff et al. 2020)

- Computational framework for population - based evaluation of TKR - implanted patellofemoral joint mechanics (Ali et al. 2020)

Design and Assessment of Patient-Specific Interventions:

- A population specific material model for sagittal craniosynostosis to predict surgical shape outcomes (Borghi et al. 2019)

- Predictive Prosthetic Socket Design. Part 1: populationbased evaluation of transtibial prosthetic sockets by FEAdriven Surrogate Modelling (Steer et al. 2019a)

- Predictive Prosthetic Socket Design. Part 2: personspecific concept design using multi-objective Genetic Algorithms (Steer et al. 2019b) 


\section{Editorial:}

The idea for this special issue on "Population based approaches to computational musculoskeletal modelling" in the journal Biomechanics and Modeling in Mechanobiology is based on a series of presentations at a symposium at the 8th World Congress of Biomechanics (WCB, 8-12 July 2018, Dublin, Ireland). In compiling this symposium, we selected studies which took a large body of anatomic, kinematic and/or device design data, produced corresponding stochastic computational models and preferably used them to investigate a clinical question or hypothesis. Thirteen papers were selected to present the state if the art in computational biomechanical analysis of musculoskeletal problems informed by population-based approaches. The authors were invited to extend their conference presentations or posters into full papers of original research, which were peer-reviewed. The research papers are accompanied by a new review article (Saxby et al. 2020), which examines the role of big data and machine learning to create physicsbased personalised models. This headline article highlights the latest ideas and encourages researchers to share models and technologies with the community.

As clinical musculoskeletal interventions and biomechanical analysis develop side by side, evidence of the need for population-based approaches has become compelling. Though the concept of patient-centred care is fundamental to our clinical partners, consideration of population variability is still not universal in biomechanical engineering research. Clinicians appreciate that challenging cases of medical device use and clinical intervention will typically involve unusual cases in the population distribution (e.g. small or large individuals; extremes of pathological or traumatic anatomy disturbance; or malpositioning of implanted devices). Furthermore, as modelling complexity builds, nonintuitive mapping between input variables and output metrics may occur, and average models may not produce results at the average of a population (Cook and Robertson 2016).

Population-based approaches offer solutions, often based on statistical shape modelling (SSM) which allows quantitative description of population variability, sometimes including tissue material characteristics. Notable applications include informing medical device geometric design, characterisation of different sub-populations and generation of virtual in silico test subjects of concern. Furthermore, anatomy can be predicted from partial data, for example estimating 3D anatomy from 2D medical images or reconstructing anatomy destroyed by trauma or tumour. Further, multivariate statistical methods such as partial least squares regression (PLSR) allow unsupervised machine learning to use medical images or anthropometric measurements to predict other biomechanical factors, such as bone fracture risk. Challenges in employing these methods concern the workload of generating large model training datasets and the computational expense of the associated simulation methods (often finite element analysis (FEA) or musculoskeletal (MSK) modelling). Furthermore, the approach requires ethical access to large, representative population anatomy datasets.

We open our collection of papers with five studies investigating Population Anatomic Analysis and Model Reconstruction. The first paper (Vallabh et al. 2019) presents an SSM of the human mandible and identifies sex-differentiating shape characteristics. They interrogated this model to identify key morphometric measurements to allow female and male shape reconstruction. A second study (Rusli and Kedgley 2019) constructs a multi-body (two-bone) SSM the first carpometacarpal (CMC) joint, at the thumb base. Their model identified variations in key joint stability influencing characteristics across their study population, which they propose may focus further research on CMC joint osteoarthritis. Three more papers move towards machine learning approaches. The first (Mei et al. 2019) presents a population comparison study employing PLSR to predict foot plantar pressure from morphometric measurements in shod and barefoot groups and makes recommendations for group-specific footwear support interventions. Expanding the concept of model generation from partial data, another paper (Davico et al. 2019) evaluates the Musculoskeletal Atlas Project (MAP) Client approach for predicting skeletal geometry for paediatric MSK modelling. They demonstrated accurate bone shape reconstruction using a variety of pipelines involving an SSM and mesh morphing informed by magnetic resonance imaging (MRI) and/or motion capture data. All MAP pipelines outperformed conventional landmark-based linear scaling. A final study (Yeung et al. 2019) aimed to predict subject-specific muscle characteristics from anthropometric measurements, comparing a range of model training methods. Balancing model simplicity with accuracy, linear PLSR was demonstrated to be effective in predicting "fat-free" muscle volume from three easily measured patient characteristics.

A second group of articles deals with bone mechanobiology, in which population-based approaches are used to predict the micro- and mesostructure of trabecular bone. In a first paper (Villette et al. 2019), structural optimisation is used to predict the trabecular bone structure from external femur cortex morphology, generated using a statistical shape model. The created trabecular bone structures showed predicted fracture risk patterns in agreement with clinical observations, including established predictors of protective and high-risk gross bone shapes. A second paper (Kirby et al. 2020) tackles the difficulty in capturing representative microstructural features in trabecular bone models by developing a probabilistic modelling framework including Voronoi cells and inverse Monte Carlo simulations. The 
generated digital bone models were compared to real trabecular bone specimens and showed good observed and statistical agreement in histomorphological parameters.

A third group of articles concerns the Design and Assessment of Standardised Orthopaedic Devices, with papers addressing total hip and knee replacement implants (THR, TKR). These are highly demanded procedures to treat conditions including osteoarthritis and articular trauma. The most commonly employed implant systems are standardised, available in a range of sizes, and a challenge in implant development is the assessment of design robustness across the range of potential recipients' anatomy and surgical variability. The first paper (Al-Dirini et al. 2019) addresses the high computational cost of precomputing training data to populate surrogate models for the assessment of surgical variability effects upon THR femoral stem stability. Surrogate models traditionally comprised a group of independent, subject-specific surrogate models, requiring many simulations to cover the full range of surgical variability. This paper presents a cohort-specific surrogate model, whereby the full variability of implant positioning scenarios is shared across the population of individuals. A second paper (Huff et al. 2020) presents a case study of population modelling to predict THR dislocation risk by feeding a finite element model with joint contact and muscle forces from musculoskeletal modelling. Surgical variation in acetabular cup position was incorporated by adjusting inclination and anteversion angles, and the model produced evidence of surgical options which might enhance dislocation resistance. Finally, a third paper (Ali et al. 2020) presents a pipeline to support the robust design of new implant systems. The computational efficiency of population analyses is particularly important in early design cycle evaluation, where many concepts are considered. The authors demonstrate a TKR case study in which adequate population variability is captured by Latin hypercube sampling for gross design screening, so that larger investments in more exhaustive Monte Carlo approaches are well selected.

Our selection of papers for this special issue concludes with a contrasting group of articles investigating Design and Assessment of Patient-Specific Interventions. First, a paper considered prediction of outcome of spring-assisted cranioplasty (SAC) surgery (Borghi et al. 2019). The authors propose that this minimally invasive cranial reshaping technique would benefit from biomechanical model-based prediction to support surgical planning and distractor spring selection, and present a population-based finite element model of SAC, using a population-derived material model for the cranioplasty callus. They found that the population-derived material model had little effect upon short-term (intraoperative) predictions, but substantially improved predictions at longer-term follow-up. Finally, two papers present the use of population approaches in personalised prosthetic socket design for rehabilitation after transtibial amputation. In the first (Steer et al. 2019a), a surrogate modelling pipeline is used to overcome the computational expense and FEA training barriers to providing prosthetists with prosthetic socket-residual limb pressure predictions. The authors present a first stochastic description of the transtibial residual limb allowing multiple individual analyses of limb-socket interactions and a $10^{6}$ times reduction in model prediction time compared to full FEA solutions. In the second (Steer et al. 2019b), the authors leverage this computationally efficient approach to investigate whether a multi-objective optimisation method might support the prosthetist in providing first-estimate socket designs and how these designs might vary depending on the optimisation objectives. This presents a first use of biomechanical modelling to propose a near-optimal prosthetic socket design, which would not replace the prosthetists' skills, but produce a first-estimate design quickly, so that they could focus their time on the high value-added detailed final stages of design.

Together, the presented papers demonstrate industry- and clinic-relevant pipelines for enhancing generic and personalised interventions and machine learning methods for efficient risk assessment for MSK problems, and present computational efficiency concepts which might address some of the shortcomings of clinically intractable patient-specific models. They go on to consider the issues associated with translating these technologies into clinical use, including presentation of information, training requirements, software/ hardware constraints, integration with the workload of busy clinicians and their concerns about deskilling.

We thank the editors of Biomechanics and Modeling in Mechanobiology for hosting this special issue. We are grateful to our volunteer peer reviewers who provided detailed and prompt appraisal of the submissions which strengthened the special issue's quality and communication. We also express our thanks to Linda Xavier for her help in managing manuscripts and making sure deadlines were met. We hope that this collection of papers will inspire future research, and enable clinician end-users to appraise it, by providing case studies illustrating the importance of considering population effects in biomechanics and toolsets to address the arising challenges.

\section{References}

Al-Dirini RMA, Martelli S, Taylor M (2019) Computational efficient method for assessing the influence of surgical variability on primary stability of a contemporary femoral stem in a cohort of subjects. Biomech Model Mechanobiol. https://doi.org/10.1007/ s10237-019-01235-0

Ali AA, Clary CW, Smoger LM, Dennis DA, Fitzpatrick CK, Rullkoetter PJ, Laz PJ (2020) Computational framework for 
population-based evaluation of TKR-implanted patellofemoral joint mechanics. Biomech Model Mechanobiol. https://doi. org/10.1007/s10237-020-01295-7

Borghi A, Florez NR, Ruggiero F, James G, O’Hara J, Ong J, Jeelani O, Dunaway D, Schievano S (2019) A population-specific material model for sagittal craniosynostosis to predict surgical shape outcomes. Biomech Model Mechanobiol. https://doi.org/10.1007/ s10237-019-01229-y

Cook DD, Robertson DJ (2016) The generic modeling fallacy: average biomechanical models often produce non-average results! J Biomech 49(15):3609-3615

Davico G, Pizzolato C, Killen BA, Barzan M, Suwarganda EK, Lloyd DG, Carty CP (2019) Best methods and data to reconstruct paediatric lower limb bones for musculoskeletal modelling. Biomech Model Mechanobiol. https://doi.org/10.1007/s10237-019-01245-y

Huff DN, Myers CA, Rullkoetter PJ (2020) Impact of alignment and kinematic variation on resistive moment and dislocation propensity for THA with lipped and neutral liners. Biomech Model Mechanobiol. https://doi.org/10.1007/s10237-020-01359-8

Kirby M, Morshed AH, Gomez J, Xiao P, Hu Y, Guo XE, Wang X (2020) Three-dimensional rendering of trabecular bone microarchitecture using a probabilistic approach. Biomech Model Mechanobiol. https://doi.org/10.1007/s10237-020-01286-8

Mei Q, Gu Y, Xiang L, Yu P, Gao Z, Shim V, Fernandez J (2019) Foot shape and plantar pressure relationships in shod and barefoot populations. Biomech Model Mechanobiol. https://doi.org/10.1007/ s10237-019-01255-w

Rusli WMR, Kedgley AE (2019) Statistical shape modelling of the first carpometacarpal joint reveals high variation in morphology. Biomech Model Mechanobiol. https://doi.org/10.1007/s1023 7-019-01257-8
Saxby DJ, Killen BA, Pizzolato C, Carty CP, Diamond LE, Modenese L, Fernandez J, Davico G, Barzan M, Lenton G, Brito da Luz S, Suwarganda E, Devaprakash D, Korhonen R, Alderson JA, Besier TF, Barrett RS, Lloyd DG (2020) Machine learning methods to support personalized neuromusculoskeletal modelling. Biomech Model Mechanobiol. https://doi.org/10.1007/s10237-020-01367-8

Steer JW, Worsley PR, Browne M, Dickinson AS (2019a) Predictive prosthetic socket design: part 1-population-based evaluation of transtibial prosthetic sockets by FEA-driven surrogate modelling. Biomech Model Mechanobiol. https://doi.org/10.1007/s1023 7-019-01195-5

Steer JW, Grudniewski PA, Browne M, Worsley PR, Sobey AJ, Dickinson AS (2019b) Predictive prosthetic socket design: part 2-generating person-specific candidate designs using multi-objective genetic algorithms. Biomech Model Mechanobiol. https://doi. org/10.1007/s10237-019-01258-7

Vallabh R, Zhang J, Fernandez J, Dimitroulis G, Ackland DC (2019) The morphology of the human mandible: a computational modelling study. Biomech Model Mechanobiol. https://doi.org/10.1007/ s10237-019-01133-5

Villette CC, Zhang J, Phillips ATM (2019) Influence of femoral external shape on internal architecture and fracture risk. Biomech Model Mechanobiol. https://doi.org/10.1007/s10237-019-01233-2

Yeung S, Fernandez JW, Handsfield GG, Walker C, Besier TF, Zhang J (2019) Rapid muscle volume prediction using anthropometric measurements and population-derived statistical models. Biomech Model Mechanobiol. https://doi.org/10.1007/s10237-019-01243-0

Publisher's Note Springer Nature remains neutral with regard to jurisdictional claims in published maps and institutional affiliations. 